Ibn Al-Haitham Jour. for Pure \& Appl. Sci. 33 (2) 2020

Ibn Al Haitham J ournal for Pure and Applied Science

J ournal homepage: http://jih.uobaghdad.edu.iq/index.php/j/index

\title{
On SAH - Ideal of BH - Algebra
}

Alaa Saleh Abed

Department of Mathematics, Faculty of Education for Girls, University of kufa, Najaf, Iraq

Alaas.abed@uokufa.edu.iq

Article history: Received 23 June 2019, Accepted 8 September 2019, Published in April

2020.

Doi: 10.30526/33.2.2433

\begin{abstract}
The aim of this investigation is to present the idea of SAH - ideal, closed SAH - ideal and closed SAH - ideal with respect to an element, $\overline{S A H}$ - ideal and s- $\overline{S A H}$ - ideal of BH algebra.

We detail and show theorems which regulate the relationship between these ideas and provide some examples in $\mathrm{BH}$ - algebra.
\end{abstract}

Keywords: $\mathrm{BH}$ - algebra, SAH - ideal of $\mathrm{BH}$ - algebra, closed $\mathrm{SAH}$ - ideal with respect to an element of $\mathrm{BH}$ - algebra, $\overline{S A H}$ - ideal .

\section{Introduction}

After founding of fuzzy subset by Zadeh L. A [1]. Several researchers presented the generalizations of the idea of fuzzy subsets. Imai and Iseki $\mathrm{K}$. established two classes BCK algebra and BCI - algebra [2, 3]. Jun Y. B., Rogh E. H. And Kin H. S. produced a new concept, named a $\mathrm{BH}$ - algebra [4]. In this paper, we will recall some basic definitions. A $\mathrm{BH}$ - algebra is a nonempty set $\Psi$ with a binary operation * satisfies the conditions: $ж * ж=0$, for all $\varkappa_{\Psi}, ж * ц=0$ and $ц * ж=0 \rightarrow ж=ц$ for all $ж, ц \in \Psi$ and $ж * 0=\%$, for all $ж \in \Psi$ [4]. we will use $\Psi$ for representing a $\mathrm{BH}$ - algebra $(\Psi ; *, 0)$. Let $\subseteq$ a nonempty subset of $\Psi$. then $\mathcal{S}$ is named an ideal of $\Psi$ if it holds: $0 \in \mathcal{S} ; \% * ц \in \mathcal{S}$ and $ц \in \mathcal{S} \rightarrow$ $ж \in \subseteq$ [4]. Let $\Psi$ and $\Phi$ be $\mathrm{BH}$ - algebras.

A mapping $\delta: \Psi \rightarrow \Phi$ is named ahomomorphism if: $\delta(ж * ц)=\delta(ж) * \delta(ц), \forall ж, Ц \in$ $\Psi$. A homomorphism $\delta$ is titled a monomerphism (resp, epimorphism) if it injective (resp., surjective). A bijective homomorphism is titled an isomorphism. Two BH - algebras $\Psi$ and $\Phi$ are said to be isomorphic, written $\Psi \cong \Phi$, if there exists an isomorphism $\delta: \Psi \rightarrow \Phi$. For any homomorphism : $\Psi \rightarrow \Phi$, the set $\left\{ж \in \Psi: \delta(ж)=0^{\prime}\right\}$ is titled the kernel of $\delta$, symbolized by $\operatorname{ker}(\delta)$, and the set $\{\delta(ж): ж \in \Psi\}$ is named the image of $\delta$, represented by $\operatorname{Im}(\delta)$. Sign that $\delta(0)=0^{\prime}, \forall$ homomorphism $\delta$ [5]. An ideal $\subseteq$ of $\Psi$ is known as closed ideal of $\Psi$ if: for each $ж \in \mathbb{S}$. 
We requisite $0 * ж \in \subseteq[6]$. Let $\subseteq$ be an ideal of $\Psi$. It is named a closed ideal with respect to an element $s \in \Psi$ (symbolized by $s-$ closed ideal) if $s *(0 * \%) \in \mathfrak{S}, \forall ж \in \mathbb{S}$ [7]. An ideal $\subseteq$ of $\Psi$ is known as completely closed ideal if $ж *\lfloor\in \subseteq, \forall, ц \in \subseteq$ [7]. Let $\subseteq$ be an ideal of $\Psi$ and $s \in \mathbb{S}$. It is named a completely closed with respect to an element $s$ (know by $\mathrm{s}-$ completely closed ideal) if: $\mathrm{s} *(\% * ц) \in \mathfrak{S}, \forall \%, ц \in \mathcal{S}$ [7]. In the next parts of our research, we will symbolize to $\mathrm{BH}-$ algebra $(€ ; *, 0)$ by $€$.

\section{Closed SAH - Ideal with Respect to an Element of BH - Algebra}

\section{Definition (1)}

An ideal $\mathfrak{V}$ of $€$ is named a SAH - ideal of $€$ if it fillfulls the requirement:

$\forall \varsigma, \zeta \in \mathfrak{V}$, if $\left(\varsigma^{*} * \zeta\right) \in \mathfrak{V}, \zeta^{*} \in \mathfrak{V} \rightarrow\left(\zeta^{*} * \varsigma\right) \in \mathfrak{V}$, where $\varsigma^{*}=\mathrm{e} * \varsigma$, and e is unit number, i.e: $\varsigma * \mathrm{e}=0$

\section{Example (2)}

Assume $€=\{0, \mathfrak{w}, \mathfrak{v}\}$ with the binary operation $*$ symbolized by the subsequent table:

Table 1.

\begin{tabular}{|c|c|c|c|}
\hline$*$ & 0 & $\mathfrak{w}$ & $\mathfrak{v}$ \\
\hline 0 & 0 & 0 & 0 \\
\hline $\mathfrak{w}$ & $\mathfrak{w}$ & 0 & 0 \\
\hline $\mathfrak{v}$ & $\mathfrak{v}$ & $\mathfrak{v}$ & 0 \\
\hline
\end{tabular}

Then the ideal $\mathfrak{Y}=\{0, \mathfrak{v}\}$ is a $\mathrm{SAH}-$ ideal of $€$.

\section{Definition (3)}

Assume $\mathfrak{Y}$ is $\mathrm{SAH}$ - ideal of $€$, then $\mathfrak{Y}$ is known as closed $\mathrm{SAH}$ - ideal if it fulfills the requirement:

$\forall \varsigma, \zeta \in \mathfrak{V}$ if $0 *\left(\varsigma^{*} * \zeta\right) \in \mathfrak{V} \wedge 0 * \zeta^{*} \in \mathfrak{V} \rightarrow 0 *\left(\zeta^{*} * \varsigma\right) \in \mathfrak{V}$

\section{Example (4)}

Assume $€=\{0,1,2,3\}$ with the binary operation $*$ definition by the ensuing table:

Table 2

\begin{tabular}{|c|c|c|c|c|}
\hline$*$ & 0 & 1 & 2 & 3 \\
\hline 0 & 0 & 1 & 0 & 0 \\
\hline 1 & 1 & 0 & 1 & 0 \\
\hline 2 & 2 & 2 & 0 & 0 \\
\hline 3 & 3 & 3 & 3 & 0 \\
\hline
\end{tabular}

Then, the ideal $\mathfrak{Y}=\{0,3\}$ is a closed SAH - ideal of $€$. 
Ibn Al-Haitham Jour. for Pure \& Appl. Sci. 33 (2) 2020

\section{Remark (5)}

We know that every SAH - ideal in $€$ is closed SAH - ideal. But the converse not correct.

\section{Example (6)}

Consider $€=\{0,1,2,3\}$ with a binary operation $*$ connoted by the ensuing table:

Table 3.

\begin{tabular}{|c|c|c|c|c|}
\hline$*$ & 0 & 1 & 2 & 3 \\
\hline 0 & 0 & 0 & 0 & 0 \\
\hline 1 & 1 & 0 & 0 & 0 \\
\hline 2 & 2 & 2 & 0 & 0 \\
\hline 3 & 3 & 2 & 2 & 0 \\
\hline
\end{tabular}

$\mathfrak{V}=\{0,3\}$ is a closed SAH - ideal of $€$ but $\mathfrak{Y}$ doesn't SAH - ideal, because:

when $\varsigma=2, \zeta=1 \rightarrow \varsigma^{*}=2, \zeta^{*}=2$

$(0 * 2=0) \in \mathfrak{V}, 0 * 2=0 \in \mathfrak{Y} \rightarrow(0 * 0=0) \in \mathfrak{Y})$, while

$(2 * 1=2) \notin \mathfrak{Y}, 2 \notin \mathfrak{V} \rightarrow(2 * 2=0) \in \mathfrak{Y}$

\section{Theorem (7)}

Assume $\left\{\mathfrak{V}_{\lambda}, \lambda \in \Lambda\right\}$ is a collocation of closed SAH - ideal of $€$. Then $\left(\bigcap_{\lambda \in \Lambda} \mathfrak{Y}_{\lambda}\right)$ is a closed $\mathrm{SAH}-$ ideal of $€$.

\section{Proof}

$\forall s, \zeta \in\left(\bigcap_{\lambda \in \Lambda} \mathfrak{V}_{\lambda}\right)$

$\therefore \varsigma, \zeta \in \mathfrak{V}_{\lambda}, \forall \lambda \in \Lambda$

$\Longrightarrow 0 *\left(\varsigma^{*} * \zeta\right) \in \mathfrak{V}_{\lambda}$ and $0 * \zeta^{*} \in \mathfrak{Y}$ then $0 *\left(\zeta^{*} * \varsigma\right) \in \mathfrak{V}_{\lambda}, \quad \forall \lambda \in \Lambda$

Since each $\mathfrak{Y}$ is closed SAH - ideal $\forall \lambda \in \Lambda$

$\Rightarrow 0 *\left(\varsigma^{*} * \zeta\right) \in\left(\bigcap_{\lambda \in \Lambda} \mathfrak{V}_{\lambda}\right)$ and $0 * \zeta^{*} \in\left(\bigcap_{\lambda \in \Lambda} \mathfrak{V}_{\lambda}\right)$ then $0 *\left(\zeta^{*} * \varsigma\right) \in\left(\bigcap_{\lambda \in \Lambda} \mathfrak{V}_{\lambda}\right)$

$\therefore\left(\bigcap_{\lambda \in \Lambda} \mathfrak{V}_{\lambda}\right)$ is closed $\mathrm{SAH}-$ ideal of $\mathrm{BH}-$ algebra $€$. 


\section{Theorem (8)}

Assume $\left\{\mathfrak{V}_{\lambda}, \lambda \in \Lambda\right\}$ is a collocation of closed SAH - ideals of $€$. Then $\left(\bigcup_{\lambda \in \Lambda} \mathfrak{V}_{\lambda}\right)$ is a closed $\mathrm{SAH}-$ ideal of $€$.

\section{Proof}

To prove that $\left(\bigcup_{\lambda \in \Lambda} \mathfrak{V}_{\lambda}\right)$ is closed SAH - ideal

$\forall s, \zeta \in\left(\bigcup_{\lambda \in \Lambda} \mathfrak{Y}_{\lambda}\right)$

$\Rightarrow \exists \mathfrak{Y}_{\mathrm{j}} \in\left\{\mathfrak{V}_{\lambda}\right\}_{\lambda \in \Lambda}$ is a c $-\mathrm{SAH}-\mathrm{ideal}$

Such that $\forall \varsigma, \zeta \in \mathfrak{Y}_{\mathrm{j}}$

$\Longrightarrow 0 *\left(\varsigma^{*} * \zeta\right) \in \mathfrak{Y}_{\mathrm{j}}$ and $0 * \zeta^{*} \in \mathfrak{Y}$ so $0 *\left(\zeta^{*} * \varsigma\right) \in \mathfrak{Y}_{\mathrm{j}}$

$\Rightarrow 0 *\left(\zeta^{*} * \varsigma\right) \in\left(\bigcup_{\lambda \in \Lambda} \mathfrak{V}_{\lambda}\right)$

$\Rightarrow\left(\bigcup_{\lambda \in \Lambda} \mathfrak{V}_{\lambda}\right)$ is closed SAH - ideal of $€$.

\section{Theorem (9)}

Assume $\left\{€_{\lambda}\right\}_{\lambda \in \Lambda}$ is a collocation of $€$ and $\mathfrak{V}_{\lambda}$ be a closed SAH - ideal of $€, \forall \lambda \in \Lambda$. Then ( $\prod_{\lambda \in \Lambda} \mathfrak{V}_{\lambda}$ ) is a closed SAH - ideal of the direct product of $€$.

\section{Proof}

$\forall\left(\mathrm{s}_{\lambda}\right),\left(\zeta_{\lambda}\right) \in \mathfrak{V}_{\lambda}$

$(0)\left(\varsigma_{\lambda}^{*}\right)\left(\zeta_{\lambda}\right) \in \prod_{\lambda \in \Lambda} \mathfrak{Y}_{\lambda} \wedge(0)\left(\zeta_{\lambda}^{*}\right) \in \prod_{\lambda \in \Lambda} \mathfrak{V}_{\lambda}$

$\Rightarrow\left(0 * \varsigma^{*} * \zeta\right) \in \prod_{\lambda \in \Lambda} \mathfrak{Y}_{\lambda} \wedge\left(0 * \zeta^{*}\right) \in \prod_{\lambda \in \Lambda} \mathfrak{V}_{\lambda}$

$0 * \varsigma^{*} * \zeta \in \mathfrak{V}_{\lambda} \wedge 0 * \zeta^{*} \in \mathfrak{V}_{\lambda}$ and

Since $\mathfrak{Y}$ is closed SAH - ideal $\forall \lambda \in \Lambda$, then

$\therefore 0 * \zeta^{*} * \varsigma \in \mathfrak{Y}_{\lambda}, \forall \lambda \in \Lambda$

$\Longrightarrow\left(0 * \zeta^{*} * \varsigma\right) \in \prod_{\lambda \in \Lambda} \mathfrak{V}_{\lambda}$

$\Longrightarrow \prod_{\lambda \in \Lambda} \mathfrak{V}_{\lambda}$ is closed SAH - ideal of $€$. 


\section{Definition (10)}

Assume $\mathfrak{Y}$ is a closed $\mathrm{SAH}$ - ideal of $€$. Then $\mathfrak{Y}$ is named closed $\mathrm{SAH}$ - ideal with respect to an element $\mathrm{s} \in €$ (represented by s -closed SAH - ideal) if:

$\mathrm{s} *\left(0 *\left(\mathrm{~s}^{*} * \zeta\right)\right) \in \mathfrak{Y} \wedge \mathrm{s} *\left(0 * \zeta^{*}\right) \in \mathfrak{Y}$. Then $\mathrm{s} *\left(0 *\left(\zeta^{*} * \varsigma\right)\right) \in \mathfrak{Y}$

\section{Example (11)}

Consider $€=\{0,1,2,3\}$ with binary operation $*$ defined by the ensuing table:

Table 4.

\begin{tabular}{|c|c|c|c|c|}
\hline$*$ & 0 & 1 & 2 & 3 \\
\hline 0 & 0 & 0 & 0 & 0 \\
\hline 1 & 1 & 0 & 0 & 1 \\
\hline 2 & 2 & 3 & 0 & 3 \\
\hline 3 & 3 & 0 & 0 & 0 \\
\hline
\end{tabular}

$\mathfrak{Y}=\{0,2\}, \mathrm{s}=3$ and $\mathfrak{Y}$ is $3-$ closed $\mathrm{SAH}-$ ideal of $€$.

\section{Completely Closed SAH - Ideal with Respect to an Element of BH - Algebra}

\section{Definition (12)}

A SAH - ideal $\mathfrak{V}$ of $€$ is known as completely closed SAH - ideal if

$\varsigma * \zeta \in \mathfrak{Y}, \forall \varsigma, \zeta \in \mathfrak{Y}$ (represented by $\overline{S A H}$-ideal).

\section{Example (13)}

In example (11), we have $\mathfrak{V}$ is $\overline{S A H}$ - ideal of $€$ since:

$0 * 0=0 \in \mathfrak{Y}, 0 * 2=0 \in \mathfrak{Y}$

$2 * 0=2 \in \mathfrak{Y}, 2 * 2=0 \in \mathfrak{Y}$

\section{Definition (14)}

A SAH - ideal $\mathfrak{Y}$ of $€$ and $s \in €$, then $\mathfrak{Y}$ is named a completely closed SAH - ideal with respect to an element $\mathrm{s} \in €$ (represented by $\mathrm{s}-\overline{S A H}$ - ideal )

If $\mathrm{s} * 0 *(\varsigma * \zeta) \in \mathfrak{Y}, \forall \varsigma, \zeta \in \mathfrak{Y}$

\section{Example (15)}

In example (11), we have:

$\mathfrak{Y}=\{0,2\}$ and $\mathrm{s}=2$, then $\mathfrak{Y}$ is $2-\overline{S A H}$ - ideal since:

$2 * 0 *(0 * 0)=2 \in \mathfrak{Y}, 2 * 0 *(0 * 2)=2 \in \mathfrak{V}$

$2 * 0 *(2 * 0)=2 \in \mathfrak{Y}, 2 * 2 *(2 * 2)=2 \in \mathfrak{Y}$

\section{Remark (16)}

In $€$ every $\mathrm{s}-\overline{S A H}$ - ideal is a s - closed SAH - ideal. 
Ibn Al-Haitham Jour. for Pure \& Appl. Sci. 33 (2) 2020

\section{Proposition (17)}

Assume $\mathfrak{Y}$ is a $\overline{S A H}$ - ideal of $€$. Then $\mathfrak{Y}$ is a $\mathrm{s}-\overline{S A H}$ - ideal, $\forall \mathrm{s} \in \mathfrak{Y}$.

\section{Proof}

Assume $\forall \varsigma, \zeta \in \mathfrak{Y}$

Mean while $\mathfrak{Y}$ is $\overline{S A H}$ - ideal and $\mathrm{s} \in \mathfrak{Y}$

Then $s * 0 *(\varsigma * \zeta) \in \mathfrak{Y}$.

\section{Theorem (18)}

Assume $(€ ; *, 0)$ and $\left(\not{L} ; \circledast, 0^{\prime}\right)$ are $\mathrm{BH}$ - algebras and $\mathfrak{h}: € \rightarrow \not \mathbb{L}$ is a $\mathrm{BH}$ - epimorphism and $\mathfrak{V}$ is a $\mathrm{SAH}$ - ideal in $€$, then $\mathfrak{h}(\mathfrak{V})$ is a SAH - ideal in $\not$.

\section{Proof}

Assume $\left(\varsigma^{*} \circledast \zeta\right) \in \mathfrak{h}(\mathfrak{Y}) \wedge \zeta^{*} \in \mathfrak{h}(\mathfrak{V})$ to prove $\left(\zeta^{*} \circledast \varsigma\right) \in \mathfrak{h}(\mathfrak{V}), \forall \varsigma, \zeta \in \mathfrak{V}$

$\Rightarrow \exists \mathrm{a}, \mathrm{b} \in \mathfrak{Y}$ such that

$\mathfrak{h}(\mathrm{a})=\mathrm{s}, \mathfrak{h}(\mathrm{b})=\zeta$,

$\left((\mathfrak{h}(\mathrm{a}))^{*} \circledast \mathfrak{h}(\mathrm{b})\right) \in \mathfrak{h}(\mathfrak{Y}) \wedge(\mathfrak{h}(\mathrm{b}))^{*} \in \mathfrak{h}(\mathfrak{V})$

$\left(\left(\mathfrak{h}(\mathrm{a})^{*} \circledast \mathfrak{h}(\mathrm{b})\right) \in \mathfrak{h}(\mathfrak{Y}) \wedge \mathfrak{h}(\mathrm{b})^{*} \in \mathfrak{h}(\mathfrak{Y})\right.$

$\mathfrak{h}\left(\mathrm{a}^{*} * \mathfrak{b}\right) \in \mathfrak{h}(\mathfrak{Y}) \wedge \mathfrak{h}\left(\mathrm{b}^{*}\right) \in \mathfrak{h}(\mathfrak{Y})$

$\Rightarrow a^{*} * b \in \mathfrak{Y} \wedge b^{*} \in \mathfrak{Y}$

$\rightarrow \mathrm{b}^{*} * \mathrm{a} \in \mathfrak{Y}$

$\rightarrow \mathfrak{h}\left(\mathrm{b}^{*} * \mathrm{a}\right) \in \mathfrak{h}(\mathfrak{Y})$

$\because \mathfrak{h}$ is epimorphism

$\Longrightarrow \mathfrak{h}\left(\mathrm{b}^{*}\right) \circledast \mathfrak{h}(\mathrm{a}) \in \mathfrak{h}(\mathfrak{Y})$

$\Rightarrow(\mathfrak{h}(\mathrm{b}))^{*} \circledast(\mathfrak{h}(\mathrm{a})) \in \mathfrak{h}(\mathfrak{Y})$

$\left(\zeta^{*} \circledast \varsigma\right) \in \mathfrak{h}(\mathfrak{Y})$

$\therefore \mathfrak{h}(\mathfrak{Y})$ is $\mathrm{SAH}-$ ideal in $\not{\ell}$.

\section{Theorem (19)}

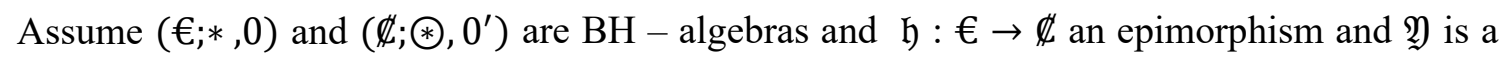
$\mathrm{SAH}$ - ideal in $€$. Then $\mathfrak{h}(\mathfrak{V})$ is a closed SAH - ideal in $\not$.

\section{Proof}

Assume $\mathfrak{Y}$ is a $\mathrm{SAH}$ - ideal in $€$

$\mathfrak{h}(\mathfrak{Y})$ is SAH - ideal (theorem (18))

And by using remark (5) 
$\mathfrak{h}(\mathfrak{Y})$ is a closed $\mathrm{SAH}-$ ideal in $\not \subset$.

\section{Remark (20)}

Now each SAH - ideal of $€$ is a $s-\overline{S A H}$ - ideal of $€, \forall s \in \mathfrak{V}$.

\section{Theorem (21)}

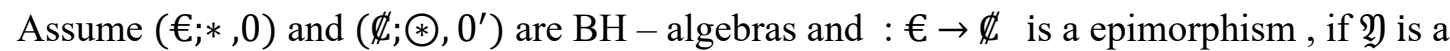
$\mathrm{s}-\overline{S A H}$ - ideal in $€$, then $\mathfrak{h}(\mathfrak{Y})$ is a $\mathfrak{h}(\mathrm{s}) \overline{S A H}$ - ideal in $\not{\not l}$.

\section{Proof}

Assume $\mathfrak{Y}$ is a $\mathrm{s}-\overline{S A H}$ - ideal in $€$, then $\mathrm{s} *(\mathrm{a} * \mathrm{c}) \in \mathfrak{Y}, \forall \mathrm{a}, \mathrm{c} \in \mathfrak{Y}$

Since $\mathfrak{Y}$ is $\mathrm{SAH}$ - ideal, then $\mathfrak{h}(\mathfrak{V})$ is a $\mathrm{SAH}$ - ideal (theorem 18)

Assume $\varsigma, \zeta \in \mathfrak{h}(\mathfrak{V})$

$\Rightarrow \exists \mathrm{m}, \mathrm{n} \in \mathfrak{Y}$ such that $\mathfrak{h}(\mathrm{m})=\varsigma, \mathfrak{h}(\mathrm{n})=\zeta$

$\mathfrak{h}(\mathrm{s}) \circledast(\mathrm{s} \circledast \mathrm{h})=\mathfrak{h}(\mathrm{s}) \circledast(\mathfrak{h}(\mathrm{m}) \circledast \mathfrak{h}(\mathrm{n}))$

$=\mathfrak{h}(\mathrm{s}) \circledast \mathfrak{h}(\mathrm{m} * \mathrm{n})$

$=\mathfrak{h}(\mathrm{s} *(\mathrm{~m} * \mathrm{n})) \in \mathfrak{h}(\mathfrak{Y})[$ since $\mathrm{s} *(\mathrm{~m} * \mathrm{n}) \in \mathfrak{V}]$

$\therefore \mathfrak{h}(\mathfrak{V})$ is a $\mathfrak{h}(\mathrm{s}) \overline{S A H}-$ ideal.

\section{Proposition (22)}

Assume $\mathfrak{Y}$ is a $\mathrm{SAH}-$ ideal of $€$ such that $\mathfrak{Y} \subseteq €_{+}$. Then $\mathfrak{Y}$ is $\mathrm{s}-\operatorname{closed} \mathrm{SAH}-$ ideal $\forall \mathrm{s} \in$ $\mathfrak{Y}$. Where $€_{+}=\{\varsigma \in €: 0 * \varsigma=0\}$.

\section{Proof}

Assume $s \in \mathfrak{V}$ and $\subseteq €_{+}$.

Then $\mathrm{s} *(0 * \varsigma)=\mathrm{s} * 0\left[\right.$ since $\left.\mathfrak{Y} \subseteq €_{+}\right]=\mathrm{s} \in \mathfrak{V}$

$\therefore \mathfrak{V}$ is $\mathrm{s}-$ closed $\mathrm{SAH}-\mathrm{ideal}$.

\section{Conclusion}

In this paper, we constructed the idea of SAH - ideal, closed SAH - ideal ,s- closed SAH - ideal , $\overline{S A H}$-ideal and s- $\overline{S A H}$ - ideal of $\mathrm{BH}$ - algebra which are presented with some of their properties, examples and theorems. In our future work, we introduce the concept of fuzzy SAH - ideal of BH - algebra. It is our optimism that this effort grows into other fundamentals for further study of ideas of BH-algebra.

\section{References}

1. Zadah, L. A. Fuzzy Sets. In form. Control.1965, 8, 338-353.

2. Iseki, K. On BCI-algebras. Mathematics Seminar Notes.1980, 8, 125-130.

3. Iseki, K.; Tanaka, S. An introduction to theory of BCK -algebras. Math. Japonica. 1978, 23, 1, 1-8.

4. Jun, Y. B.; Roh, E.H. On BH-algebra. scientiae Mathematica.1998, 1, 1, 347-354. 
Ibn Al-Haitham Jour. for Pure \& Appl. Sci. 33 (2) 2020

5. Jun, Y.B.; Kim, H.S.; Kondo, M. On BH-relations in BH algebras. Scientiae

6. Baik, H.G. On Vague BH -subalgebra of BH- algebras. International Mathematical Forum.2009, 4, 17, 823-829.

7. Abass, H.H.; Dahham, H. A. On Completely Closed Ideal With Respect To An Element Of A BH-Algebra. Journal of Karbala university.2012, 10, 3, 302-312. 